Supporting Information

\title{
Aluminacyclopropene: Syntheses, Characterization, and Reactivity toward Terminal Alkynes
}

Hongping Zhu, ${ }^{\dagger}$ Rainer B. Oswald, ${ }^{\dagger}$ Hongjun Fan, ${ }^{\S}$ Herbert W. Roesky, ${ }^{\dagger *}$ Qingjun Ma, ${ }^{\dagger}$ Zhi Yang, ${ }^{\dagger}$ Hans-Georg Schmidt, ${ }^{\dagger}$ Mathias Noltemeyer, ${ }^{\dagger}$ Kerstin Starke, ${ }^{\dagger}$ and Narayan S. Hosmane $e^{\xi}$

${ }^{\dagger}$ Institut für Anorganische Chemie der Universität Göttingen, Tammannstrasse 4, D37077 Göttingen, Germany $\stackrel{\ddagger}{\ddagger}$ stitut für Physikalische Chemie der Universität Göttingen, Tammannstrasse 6, D-37077 Göttingen, Germany ${ }^{\S}$ Labor für Physikalische und Theoretische Chemie, Universität Siegen, 57068 Siegen, Germany ${ }^{\xi}$ Department of Chemistry and Biochemistry, Northern Illinois University, Dekalb, Illinois 60115-2862 


\section{The selected computational results}

(1). The table lists the bonds ( $\mathrm{Al}-\mathrm{C}$ and $\mathrm{C}-\mathrm{C}$ (in the $\mathrm{AlC}_{2}$ ring) and $\mathrm{Al}-\mathrm{N}$ ) situation followed by the analysis values of the related atom valence and bond order.

(2). The Hartree energy values and the corresponding $\mathrm{Al}-\eta^{2}-\mathrm{C}_{2}$ bond dissociation energy estimated according to $\Delta \mathrm{E}=\mathrm{E}\left(\mathrm{LAl}+\mathrm{R}^{1} \mathrm{C} \equiv \mathrm{CR}^{2}\right)-\mathrm{E}\left(\mathrm{LAl}\left(\eta^{2}-\mathrm{C}_{2} \mathrm{R}^{1} \mathrm{R}^{2}\right)(\mathrm{L}=\right.$ $\left.\mathrm{HC}[(\mathrm{CMe})(\mathrm{NAr})]_{2}, \mathrm{Ar}=2,6-i \mathrm{Pr}_{2} \mathrm{C}_{6} \mathrm{H}_{3}\right)$.

(a) for compound $\mathrm{LAl}\left(\eta^{2}-\mathrm{C}_{2} \mathrm{H}_{2}\right)$,<smiles></smiles>

\begin{tabular}{|c|c|c|c|c|c|c|c|c|c|c|}
\hline Bond & Occ. & MO 1 & $\begin{array}{l}\text { Contr. } \\
{[\%]}\end{array}$ & $\begin{array}{l}\text { Type } \\
\text { (contr. [\%]) }\end{array}$ & MO 2 & $\begin{array}{l}\text { Contr. } \\
{[\%]}\end{array}$ & $\begin{array}{l}\text { Type } \\
\text { (contr. [\%]) }\end{array}$ & MO 3 & $\begin{array}{l}\text { Contr. } \\
{[\%]}\end{array}$ & $\begin{array}{l}\text { Type } \\
\text { (contr.[\%]) }\end{array}$ \\
\hline $\mathrm{Al}(27)-\mathrm{C}(28)$ & 1.94358 & $\mathrm{Al}(27)$ & 20.12 & $\begin{array}{l}\mathrm{s}(25.71) \\
\mathrm{p}^{2.86}(73.42)\end{array}$ & $\mathrm{C}(28)$ & 79.88 & $\begin{array}{l}\mathrm{s}(30.55) \\
\mathrm{p}^{2.27}(69.41)\end{array}$ & & & \\
\hline $\mathrm{Al}(27)-\mathrm{C}(29)$ & 1.94364 & $\operatorname{Al}(27)$ & 20.13 & $\begin{array}{l}\mathrm{s}(25.72) \\
\mathrm{p}^{2.85}(73.41)\end{array}$ & $\mathrm{C}(29)$ & 79.87 & $\begin{array}{l}\mathrm{s}(30.55) \\
\mathrm{p}^{2.27}(69.42)\end{array}$ & & & \\
\hline $\mathrm{C}(28)-\mathrm{C}(29)$ & 1.98446 & $\mathrm{C}(28)$ & 50.00 & $\begin{array}{l}\mathrm{s}(35.00) \\
\mathrm{p}^{1.86(}(64.94)\end{array}$ & $\mathrm{C}(29)$ & 50.00 & $\begin{array}{l}\mathrm{s}(35.00) \\
\mathrm{p}^{1.86(64.94)}\end{array}$ & & & \\
\hline $\begin{array}{l}\mathrm{Al}(27)- \\
\mathrm{C}(28)-\mathrm{C}(29)\end{array}$ & 1.96889 & $\mathrm{Al}(27)$ & 4.22 & $\begin{array}{l}\mathrm{p}^{1.00}(63.01) \\
\mathrm{d}^{0.59}(36.99)\end{array}$ & $\mathrm{C}(28)$ & 47.89 & $\mathrm{p}^{1.00}(99.83)$ & $\mathrm{C}(29)$ & 47.89 & $\mathrm{p}^{1.00}(99.83)$ \\
\hline $\mathrm{Al}(27)-\mathrm{N}(12)$ & 1.90530 & $\mathrm{~N}(12)$ & 92.49 & $\begin{array}{l}\mathrm{s}(33.12) \\
\mathrm{p}^{2.02}(66.88)\end{array}$ & $\mathrm{Al}(27)$ & 7.51 & $\begin{array}{l}\mathrm{s}(24.26) \\
\mathrm{p}^{1.82}(44.20) \\
\mathrm{d}^{1.30}(31.54)\end{array}$ & & & \\
\hline $\mathrm{Al}(27)-\mathrm{N}(16)$ & 1.90530 & $\mathrm{~N}(16)$ & 92.49 & $\begin{array}{l}\mathrm{s}(33.12) \\
\mathrm{p}^{2.02}(66.88)\end{array}$ & $\mathrm{Al}(27)$ & 7.51 & $\begin{array}{l}\mathrm{s}(24.26) \\
\mathrm{p}^{1.82}(44.20) \\
\mathrm{d}^{1.30}(31.54)\end{array}$ & & & \\
\hline
\end{tabular}

Occ. $=$ Occupancy; Contr. $=$ Contribution 
Atom Valence:

Al 3.66287

N 3.05096, N 3.05097

C 3.72569, C 3.72570

Bond Order:

Al-N 0.6102, 0.6102

Al-C 1.1028, 1.1030

C-C 1.6665

the corresponding $\mathrm{Al}-\eta^{2}-\mathrm{C}_{2}$ bond dissociation energy

Total energy (in Hartree): - 1480.57325283

LAl energy (in Hartree): -1403.19114639

$\mathrm{C}_{2} \mathrm{H}_{2}$ Fragment energy (in Hartree): -77.3279533487

Dissociation energy: $142.18 \mathrm{~kJ} / \mathrm{mol}$

(b) for compound $\mathrm{LAl}\left[\eta^{2}-\mathrm{C}_{2}(\mathrm{H})(\mathrm{Ph})\right]$,<smiles></smiles>

\begin{tabular}{|l|l|l|l|l|l|l|l|}
\hline Bond & Occ. & MO 1 & $\begin{array}{l}\text { Contr. } \\
{[\%]}\end{array}$ & $\begin{array}{l}\text { Type } \\
\text { (contr. [\%] })\end{array}$ & MO 2 & $\begin{array}{l}\text { Contr. } \\
{[\%]}\end{array}$ & $\begin{array}{l}\text { Type } \\
\text { (contr. [\%] })\end{array}$ \\
\hline $\mathrm{Al}(27)-\mathrm{C}(28)$ & 1.95106 & $\mathrm{Al}(27)$ & 19.88 & $\begin{array}{l}\mathrm{s}(37.03) \\
\mathrm{p}^{1.68}(62.21)\end{array}$ & $\mathrm{C}(28)$ & 80.12 & $\mathrm{~s}(31.01)$ \\
& & & & $\mathrm{s}(40.01)$ & $\mathrm{C}(29)$ & 79.56 & $\mathrm{~s}(26.99)$ \\
\hline $\mathrm{Al}(27)-\mathrm{C}(29)$ & 1.93546 & $\mathrm{Al}(27)$ & 20.44 & & & \\
\hline
\end{tabular}




\begin{tabular}{|c|c|c|c|c|c|c|c|}
\hline & & & & $\mathrm{p}^{1.48}(59.30)$ & & & $\mathrm{p}^{2.70}(72.99)$ \\
\hline $\mathrm{C}(28)-\mathrm{C}(29)$ & 1.97682 & $\mathrm{C}(28)$ & 48.81 & $\begin{array}{l}\mathrm{s}(34.96) \\
\mathrm{p}^{1.86}(64.97)\end{array}$ & $\mathrm{C}(29)$ & 51.19 & $\begin{array}{l}\mathrm{s}(35.69) \\
\mathrm{p}^{1.80}(64.26)\end{array}$ \\
\hline $\mathrm{C}(28)-\mathrm{C}(29)$ & 1.84945 & $\mathrm{C}(28)$ & 49.76 & $\mathrm{p}^{1.00}(99.84)$ & $\mathrm{C}(29)$ & 50.24 & $\mathrm{P}^{1.00}(99.87)$ \\
\hline $\mathrm{Al}(27)-\mathrm{N}(12)$ & 1.90609 & $\mathrm{~N}(12)$ & 90.75 & $\begin{array}{l}\mathrm{s}(29.33) \\
\mathrm{p}^{2.41}(70.67)\end{array}$ & $\mathrm{Al}(27)$ & 9.25 & $\begin{array}{l}\mathrm{s}(26.42) \\
\mathrm{p}^{2.71}(71.48) \\
\mathrm{d}^{0.08}(2.10)\end{array}$ \\
\hline $\mathrm{Al}(27)-\mathrm{N}(16)$ & 1.90609 & $\mathrm{~N}(16)$ & 90.75 & $\begin{array}{l}\mathrm{s}(29.33) \\
\mathrm{p}^{2.41}(70.67)\end{array}$ & $\mathrm{Al}(27)$ & 9.25 & $\begin{array}{l}\mathrm{s}(26.42) \\
\mathrm{p}^{2.71}(71.48) \\
\mathrm{d}^{0.08}(2.10)\end{array}$ \\
\hline
\end{tabular}

Occ. $=$ Occupancy; Contr. $=$ Contribution

Atom Valence:

Al 3.64694

N 3.0515, N 3.05165

C 3.71245, C 3.66683

Bond Order:

Al-N 0.6123, 0.6124

Al-C 1.0825, 1.0756

C-C 1.5728

the corresponding $\mathrm{Al}-\eta^{2}-\mathrm{C}_{2}$ bond dissociation energy

Total energy (in Hartree): -1711.64098282

LAl energy (in Hartree): -1403.19114639

HCCPh Fragment energy (in Hartree): -308.398702865

Dissociation energy: $134.25 \mathrm{~kJ} / \mathrm{mol}$ 
(c) for compound $\mathrm{LAl}\left(\eta^{2}-\mathrm{C}_{2} \mathrm{Ph}_{2}\right)$,<smiles>CC1=CC(C)=[N+]([Al])[Al](N([Al])[Al])C1=Cc1ccccc1</smiles>

\begin{tabular}{|c|c|c|c|c|c|c|c|}
\hline Bond & Occ. & MO 1 & $\begin{array}{l}\text { Contr. } \\
{[\%]}\end{array}$ & $\begin{array}{l}\text { Type } \\
\text { (contr. [\%]) }\end{array}$ & MO 2 & $\begin{array}{l}\text { Contr. } \\
\text { [\%] }\end{array}$ & $\begin{array}{l}\text { Type } \\
\text { (contr. [\%]) }\end{array}$ \\
\hline $\mathrm{Al}(27)-\mathrm{C}(28)$ & 1.92021 & $\mathrm{Al}(27)$ & 19.64 & $\begin{array}{l}\mathrm{s}(39.39) \\
\mathrm{p}^{1.52}(59.96)\end{array}$ & $\mathrm{C}(28)$ & 80.36 & $\begin{array}{l}\mathrm{s}(26.21) \\
\mathrm{p}^{2.81}(73.76)\end{array}$ \\
\hline $\mathrm{Al}(27)-\mathrm{C}(29)$ & 1.91492 & $\operatorname{Al}(27)$ & 19.43 & $\begin{array}{l}\mathrm{s}(38.73) \\
\mathrm{p}^{1.57}(60.61)\end{array}$ & $\mathrm{C}(29)$ & 80.57 & $\begin{array}{l}\mathrm{s}(26.11) \\
\mathrm{p}^{2.83}(73.87)\end{array}$ \\
\hline $\mathrm{C}(28)-\mathrm{C}(29)$ & 1.96360 & $\mathrm{C}(28)$ & 50.02 & $\begin{array}{l}\mathrm{s}(36.29) \\
\mathrm{p}^{1.75}(63.66)\end{array}$ & $\mathrm{C}(29)$ & 49.98 & $\begin{array}{l}\mathrm{s}(36.42) \\
\mathrm{p}^{1.74}(63.53)\end{array}$ \\
\hline $\mathrm{C}(28)-\mathrm{C}(29)$ & 1.83372 & $\mathrm{C}(28)$ & 50.04 & $\begin{array}{l}\mathrm{s}(0.08) \\
\mathrm{p}^{99.99}(99.80)\end{array}$ & $\mathrm{C}(29)$ & 49.96 & $\begin{array}{l}\mathrm{s}(0.11) \\
\mathrm{p}^{99.99}(99.77)\end{array}$ \\
\hline $\mathrm{Al}(27)-\mathrm{N}(12)$ & 1.94033 & $\mathrm{~N}(12)$ & 90.73 & $\begin{array}{l}\mathrm{s}(29.37) \\
\mathrm{p}^{2.41}(70.63)\end{array}$ & $\operatorname{Al}(27)$ & 9.27 & $\begin{array}{l}\mathrm{s}(26.40) \\
\mathrm{p}^{2.71}(71.48) \\
\mathrm{d}^{0.08}(2.08)\end{array}$ \\
\hline $\mathrm{Al}(27)-\mathrm{N}(16)$ & 1.94050 & $\mathrm{~N}(16)$ & 90.75 & $\begin{array}{l}\mathrm{s}(29.36) \\
\mathrm{p}^{2.41}(70.64)\end{array}$ & $\mathrm{Al}(27)$ & 9.25 & $\begin{array}{l}\mathrm{s}(26.52) \\
\mathrm{p}^{2.69}(71.39) \\
\mathrm{d}^{0.08}(2.09)\end{array}$ \\
\hline
\end{tabular}

Occ. $=$ Occupancy; Contr. $=$ Contribution

Atom Valence:

Al 3.6213

N 3.04857, N 3.0488

C 3.66378, C 3.64842

Bond Order: 
Al-N 0.6153, 0.6137

Al-C 1.0579, 1.0366

C-C 1.5180

the corresponding $\mathrm{Al}-\eta^{2}-\mathrm{C}_{2}$ bond dissociation energy

Total energy (in Hartree): -1942.70246756

LAl energy (in Hartree): -1403.19114639

PhCCPh Fragment energy (in Hartree) : -539.470239472

Dissociation energy: $107.86 \mathrm{~kJ} / \mathrm{mol}$

(d) for compound $\mathrm{LAl}\left[\eta^{2}-\mathrm{C}_{2}\left(\mathrm{SiMe}_{3}\right)_{2}\right]$,

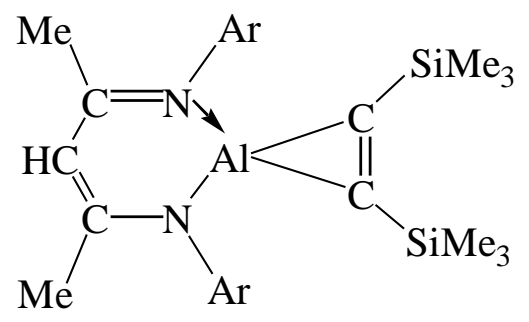

\begin{tabular}{|c|c|c|c|c|c|c|c|c|c|c|}
\hline Bond & Occ. & MO 1 & $\begin{array}{l}\text { Contr. } \\
{[\%]}\end{array}$ & $\begin{array}{l}\text { Type } \\
\text { (contr. [\%]) }\end{array}$ & MO 2 & $\begin{array}{l}\text { Contr. } \\
{[\%]}\end{array}$ & $\begin{array}{l}\text { Type } \\
\text { (contr. [\%]) }\end{array}$ & MO 3 & $\begin{array}{l}\text { Contr. } \\
{[\%]}\end{array}$ & $\begin{array}{l}\text { Type } \\
\text { (contr. [\%]) }\end{array}$ \\
\hline $\mathrm{Al}(27)-\mathrm{C}(28)$ & 1.91815 & $\mathrm{Al}(27)$ & 18.98 & $\begin{array}{l}\mathrm{s}(25.38) \\
\mathrm{p}^{2.90}(73.70)\end{array}$ & $\mathrm{C}(28)$ & 81.02 & $\begin{array}{l}\mathrm{s}(23.00) \\
\mathrm{p}^{3.35}(76.97)\end{array}$ & & & \\
\hline $\mathrm{Al}(27)-\mathrm{C}(29)$ & 1.91810 & $\mathrm{Al}(27)$ & 18.92 & $\begin{array}{l}\mathrm{s}(25.29) \\
\mathrm{p}^{2.92}(73.83)\end{array}$ & $\mathrm{C}(29)$ & 81.08 & $\begin{array}{l}\mathrm{s}(23.01) \\
\mathrm{p}^{3.34}(76.96)\end{array}$ & & & \\
\hline $\mathrm{C}(28)-\mathrm{C}(29)$ & 1.97600 & $\mathrm{C}(28)$ & 50.01 & $\begin{array}{l}\mathrm{s}(35.10) \\
\mathrm{p}^{1.85}(64.84)\end{array}$ & $\mathrm{C}(29)$ & 49.99 & $\begin{array}{l}\mathrm{s}(35.08) \\
\mathrm{p}^{1.85}(64.87)\end{array}$ & & & \\
\hline $\begin{array}{l}\mathrm{Al}(27)- \\
\mathrm{C}(28)-\mathrm{C}(29)\end{array}$ & 1.93605 & $\mathrm{Al}(27)$ & 3.53 & $\begin{array}{l}\mathrm{p}^{1.00}(62.79) \\
\mathrm{d}^{0.59}(37.21)\end{array}$ & $\mathrm{C}(28)$ & 48.21 & $\mathrm{p}^{1.00}(99.88)$ & $\mathrm{C}(29)$ & 48.26 & $\mathrm{p}^{1.00}(99.88)$ \\
\hline $\mathrm{Al}(27)-\mathrm{N}(12)$ & 1.90609 & $\mathrm{~N}(12)$ & 92.46 & $\mathrm{~s}(32.97)$ & $\mathrm{Al}(27)$ & 7.54 & $\mathrm{~s}(24.59)$ & & & \\
\hline
\end{tabular}




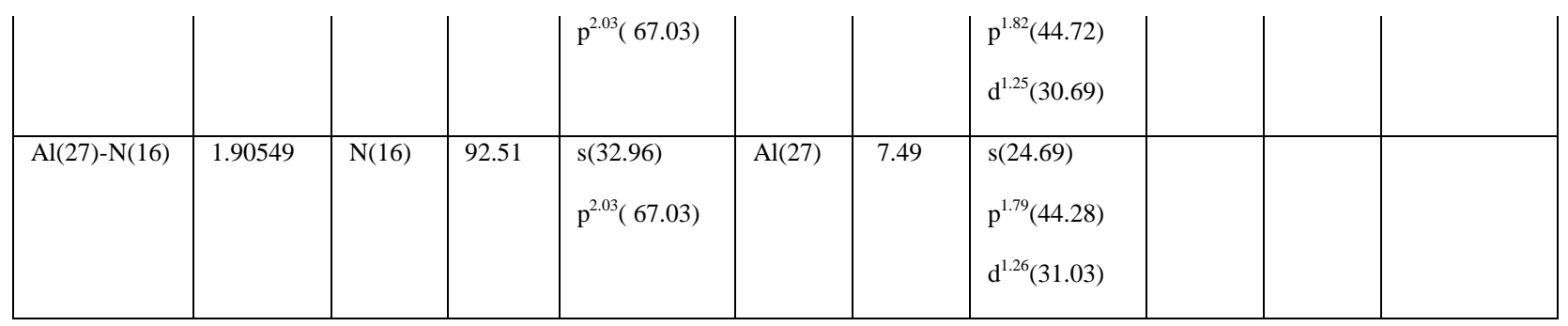

Occ. $=$ Occupancy; Contr. $=$ Contribution

Atom Valence:

Al 3.63956

N 3.04343, N 3.04519

C 3.58774, C 3.58915

Bond Order:

Al-N 0.6127, 0.6121

Al-C 1.0304, 1.0320

C-C 1.4832

the corresponding $\mathrm{Al}-\eta^{2}-\mathrm{C}_{2}$ bond dissociation energy

Total energy (in Hartree): -2297.95163585

LAl-energy (in Hartree): -1403.19114639

$\mathrm{Me}_{3} \mathrm{SiCCSiMe}_{3}$ Fragment energy (in Hartree): -894.735022179

Dissociation energy: $82.62 \mathrm{~kJ} / \mathrm{mol}$

(e) for compound $\mathrm{LAl}\left[\eta^{2}-\mathrm{C}_{2}\left(\mathrm{SiMe}_{3}\right)\left(\mathrm{CCSiMe}_{3}\right)\right]$, 


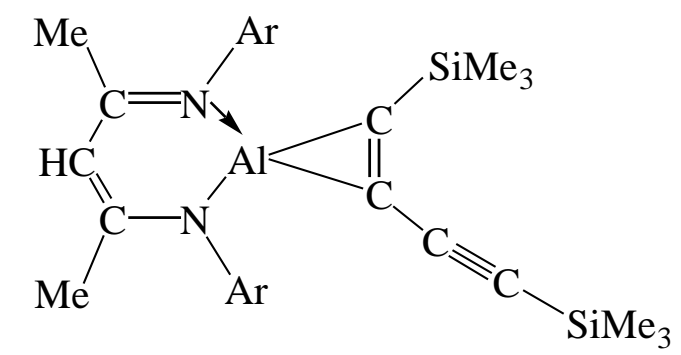

\begin{tabular}{|c|c|c|c|c|c|c|c|}
\hline Bond & Occ. & MO 1 & $\begin{array}{l}\text { Contr. } \\
{[\%]}\end{array}$ & $\begin{array}{l}\text { Type } \\
\text { (contr. [\%]) }\end{array}$ & MO 2 & $\begin{array}{l}\text { Contr. } \\
{[\%]}\end{array}$ & $\begin{array}{l}\text { Type } \\
\text { (contr. [\%]) }\end{array}$ \\
\hline $\mathrm{Al}(27)-\mathrm{C}(28)$ & 1.93896 & $\mathrm{Al}(27)$ & 19.37 & $\begin{array}{l}\mathrm{s}(40.10) \\
\mathrm{p}^{1.48}(59.17)\end{array}$ & $\mathrm{C}(28)$ & 80.63 & $\begin{array}{l}\mathrm{s}(25.20) \\
\mathrm{p}^{2.97}(74.76)\end{array}$ \\
\hline $\mathrm{Al}(27)-\mathrm{C}(29)$ & 1.88500 & $\mathrm{Al}(27)$ & 17.86 & $\begin{array}{l}\mathrm{s}(37.42) \\
\mathrm{p}^{1.65}(61.85)\end{array}$ & $\mathrm{C}(29)$ & 82.14 & $\begin{array}{l}\mathrm{s}(26.12) \\
\mathrm{p}^{2.83}(73.87)\end{array}$ \\
\hline $\mathrm{C}(28)-\mathrm{C}(29)$ & 1.96149 & $\mathrm{C}(28)$ & 48.78 & $\begin{array}{l}\mathrm{s}(32.09) \\
\mathrm{p}^{2.11}(67.84)\end{array}$ & $\mathrm{C}(29)$ & 51.22 & $\begin{array}{l}\mathrm{s}(37.86) \\
\mathrm{p}^{1.64}(62.11)\end{array}$ \\
\hline $\mathrm{C}(28)-\mathrm{C}(29)$ & 1.83372 & $\mathrm{C}(28)$ & 49.56 & $\mathrm{p}^{1.00}(99.87)$ & $C(29)$ & 50.44 & $\mathrm{p}^{1.00}(99.89)$ \\
\hline $\mathrm{Al}(27)-\mathrm{N}(12)$ & 1.94081 & $\mathrm{~N}(12)$ & 90.72 & $\begin{array}{l}\mathrm{s}(29.24) \\
\mathrm{p}^{2.42}(70.75)\end{array}$ & $\operatorname{Al}(27)$ & 9.28 & $\begin{array}{l}\mathrm{s}(26.93) \\
\mathrm{p}^{2.64}(70.97) \\
\mathrm{d}^{0.08}(2.10)\end{array}$ \\
\hline $\mathrm{Al}(27)-\mathrm{N}(16)$ & 1.94080 & $\mathrm{~N}(16)$ & 90.72 & $\begin{array}{l}\mathrm{s}(29.23) \\
\mathrm{p}^{2.42}(70.77)\end{array}$ & $\operatorname{Al}(27)$ & 9.28 & $\begin{array}{l}\mathrm{s}(26.94) \\
\mathrm{p}^{2.63}(70.96) \\
\mathrm{d}^{0.08}(2.10)\end{array}$ \\
\hline
\end{tabular}

Occ. $=$ Occupancy; Contr. $=$ Contribution

Atom Valence:

Al 3.63420

N 3.05656, N 3.05636

C 3.59725 , C 3.40562

Bond Order: 
Al-N 0.6232, 0.6241

Al-C 1.0625, 1.0043

C-C 1.4357

the corresponding $\mathrm{Al}-\eta^{2}-\mathrm{C}_{2}$ bond dissociation energy

Total energy (in Hartree): -2374.13278808

LAl energy (in Hartree): -1403.19114639

$\mathrm{Me}_{3} \mathrm{SiCC}_{\mathrm{CCCSiMe}}$ Fragment energy (in Hartree): -970.906550926

Dissociation energy: $92.13 \mathrm{~kJ} / \mathrm{mol}$

\section{The detailed reference}

reference (16):

Gaussian 03, Revision C.02. Frisch, M. J.; Trucks, G. W.; Schlegel, H. B.; Scuseria, G. E.; Robb, M. A.; Cheeseman, J. R.; Montgomery, J. A.; Jr.; Vreven, T.; Kudin, K. N.; Burant, J. C.; Millam, J. M.; Iyengar, S. S.; Tomasi, J.; Barone, V.; Mennucci, B.; Cossi, M.; Scalmani, G.; Rega, N.; Petersson, G. A.; Nakatsuji, H.; Hada, M.; Ehara, M.; Toyota, K.; Fukuda, R.; Hasegawa, J.; Ishida, M.; Nakajima, T.; Honda, Y.; Kitao, O.; Nakai, H.; Klene, M.; Li, X.; Knox, J. E.; Hratchian, H. P.; Cross, J. B.; Bakken, V.; Adamo, C.; Jaramillo, J.; Gomperts, R.; Stratmann, R. E.; Yazyev, O.; Austin, A. J.; Cammi, R.; Pomelli, C.; Ochterski, J. W.; Ayala, P. Y.; Morokuma, K.; Voth, G. A.; Salvador, P.; Dannenberg, J. J.; Zakrzewski, V. G.; Dapprich, S.; Daniels, A. D.; Strain, M. C.; Farkas, O.; Malick, D. K.; Rabuck, A. D.; Raghavachari, K.; Foresman, J. B.; Ortiz, J. V.; Cui, Q.; Baboul, A. G.; Clifford, S.; Cioslowski, J.; Stefanov, B. B.; 
Liu, G.; Liashenko, A.; Piskorz, P.; Komaromi, I.; Martin, R. L.; Fox, D. J.; Keith, T.; Al-Laham, M. A.; Peng, C. Y.; Nanayakkara, A.; Challacombe, M.; Gill, P. M. W.; Johnson, B.; Chen, W.; Wong, M. W.; Gonzalez, C.; Pople, J. A. Gaussian, Inc., Wallingford CT, 2004.

reference (20):

Ahlrichs, R.; Bär, M.; Baron, H.-P.; Bauernschmitt, R.; Böcker, S.; Deglmann, P.; Ehrig, M.; Eichkorn, K.; Elliott, S.; Furche, F.; Haase, F.; Häser, M.; Horn, H.; Hättig, C.; Huber, C.; Huniar, U.; Kattannek, M.; Köhn, A.; Kölmel, C.; Kollwitz, M.; May, K.; Ochsenfeld, C.; Öhm, H.; Schäfer, A.; Schneider, U.; Sierka, M.; Treutler, O.; Unterreiner, B.; Arnim, M. V.; Weigand, F.; Weis, P.; Weiss, H. TURBOMOLE 5.5, University of Karlsruhe, Germany, 2002. 